\title{
A case series of hematohidrosis: A puzzling medical phenomenon
}

\author{
Elham Shahgholi \\ Division of Pediatric Hematology Oncology, Bahrami Children Hospital, Tehran University of Medical Sciences, Tehran, \\ Iran.Email: eshahgholi@gmail.com \\ Received: 31st October 2017, Revised: 16th December 2017, Accepted: 30th December 2017
}

SUMMARY: Shahgholi E. A case series of hematohidrosis: A puzzling medical phenomenon. Turk J Pediatr 2018; 60: 757-761.

Hematohidrosis (bloody sweat), also known as hematidrosis, is a rare clinical entity characterized by recurrent, spontaneous, self-limited episodes of blood oozing from intact skin or mucosa that are witnessed by medical personnel. We hereby report three healthy children with the history of recurrent episodes of bleeding from the intact skin over their various parts of the body. Most of these bleeding episodes occurred after stressful situations. On the basis of clinical presentation and normal investigation, they were diagnosed with hematohidrosis. We treated them with non-specific beta-blocker (propranolol). Follow-up observation of these patients demonstrated marked diminish in the frequency and severity of their episodes. In spite of the rarity of hematohidrosis, it should be considered as a differential diagnosis of bleeding episodes in patients with normal physical and laboratory investigations.

Key words: hematohidrosis, bleeding episode, beta-blocker.

Hematohidrosis (bloody sweat), also known as hematidrosis, is a rare disorder, with only a few cases reported to date. It is characterized by exudation of bloody secretion from intact skin and mucosa. This phenomenon has been mostly reported during extreme bouts of fear or anxiety. Each episode is usually self-limiting. Physical and laboratory investigations do not lead to any abnormal results, making it difficult to fully understand this phenomenon. ${ }^{1}$ Herein we present three cases of this rare condition and discuss possible mechanisms to explain their clinical manifestation.

\section{Case Reports}

Three cases of hematohidrosis, including two girls (aged 9 and 11years) and one boy (aged 8 years) are reported. The second and third cases were diagnosed similarly over a period of three years following the diagnosis of hematohydrosis in the first case. Informed consent forms were signed by parents of all children.

\section{Case 1}

An 11-year-old pre-pubertal girl presented to our hematology clinic with the history of bleeding from the intact skin over the forehead, eyes, ears, nails, arm, umbilical area, back, vagina, and gastrointestinal tract for several months. Her family revealed that these episodes happened following extreme physical or emotional stress but sometimes occurred spontaneously without any initiating factor or during sleep. The bleeding was spontaneous, recurrent and painless and subsided as soon as the site was wiped. It occurred in episodes, once or twice a day, sometimes more frequently. Most of these episodes were preceded by a brief period of abdominal pain, vomiting or a headache. Each episode started with bubbly, viscous bright-red colored bloody secretion in a limited area of her body, lasted for about 10-15 minutes and ceased spontaneously.

She was thoroughly investigated for all type of bleeding disorders but no demonstrable hematological problem was detected. Upper gastrointestinal endoscopy and ultrasound of the abdomen did not reveal any abnormality. All dermatology, ophthalmology and psychology consults returned inconclusive and no cause was speculated. The patient was admitted to the hospital and carefully observed to rule 
out child abuse and malingering. During her admission, two episodes occurred under our visual monitoring. In each episode we observed a moist, viscous, bubbly blood stained sweaty discharge in a limited area of her forearm (Fig. 1a) and back (Fig. 1b). The skin over the affected area was fully examined but no lesion was observed. After wiping the area, there was no evidence of oozing. The patient exhibited no distress during her bleeding episodes and cooperated well. Smears of the bloody exudate contained all of the components of peripheral blood. On the basis of her clinical presentation,
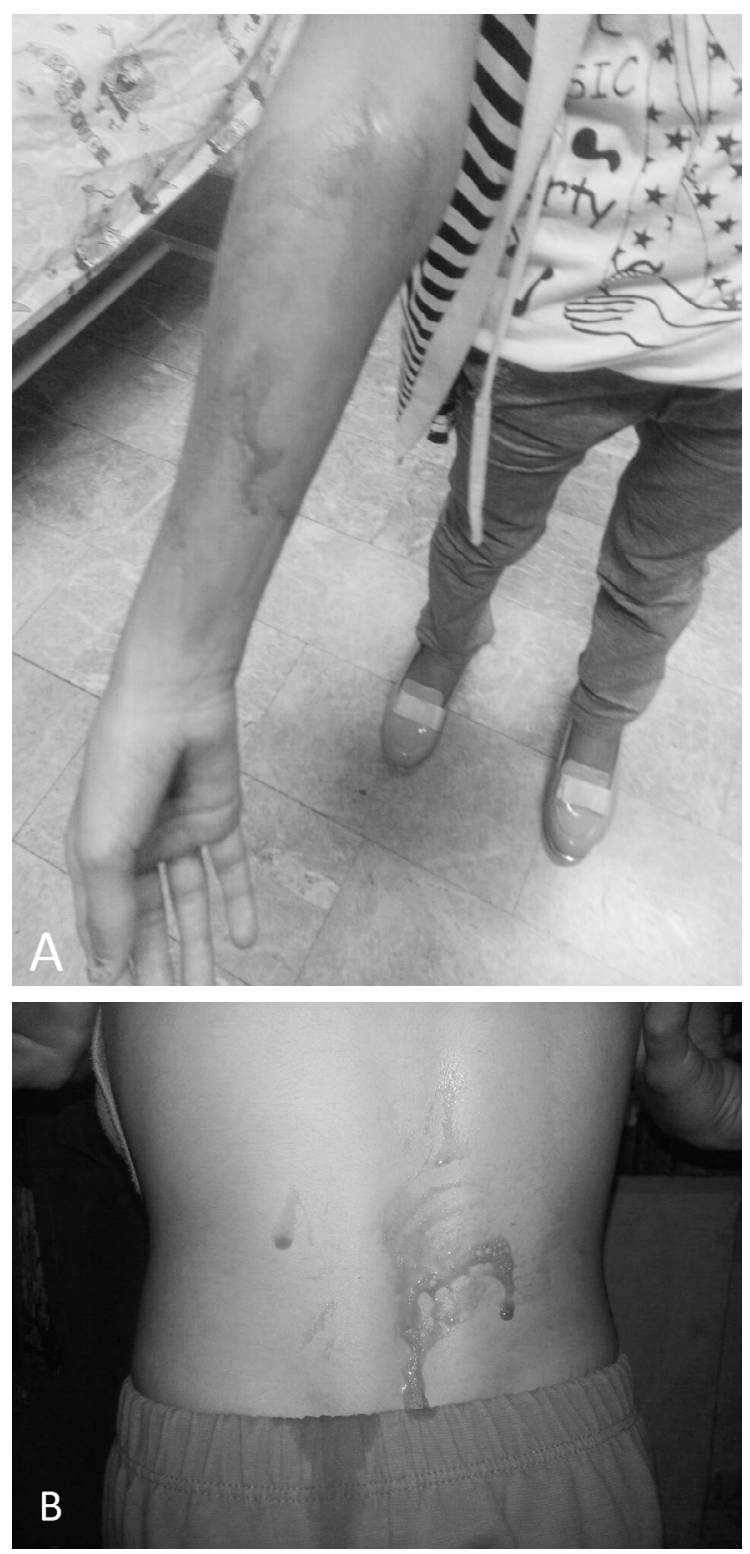

Fig. 1. Blood stained sweaty discharge from a) the forearm and b) back of an 11-year-old pre-pubertal girl. diagnosis of hematohidrosis was made. Apart from regular counseling for anxiety disorder, we treated her with beta-blocker (propranolol $10 \mathrm{mg}$ daily). During six months follow-up, she had demonstrated marked improvement and the frequency and severity of her episodes diminished significantly but did not completely disappear.

\section{Case 2}

An 8-year-old boy who was the only child of a high socioeconomic family was referred to our clinic for painless, spontaneous bleeding from the nail beds of his hands and feet. According to his mother, the oozing of bloody secretion from his fingers were preceded by issues such as an upcoming exam, playing a computer game, watching fiction TV movies or when parents not satisfying his demands (Fig. 2). His mother was very concerned about the seriousness of his son's illness and consulted many medical professionals but no specific diagnosis was made. All physical and laboratory investigations were within normal limits. The diagnosis of hematohidrosis was confirmed by medical personnel who observed the bleeding. Survey of photographs of these bleeding episodes convinced us the diagnosis of hematohidrosis. Parents were provided information about the benign and self-limited nature of the illness. After 2-year follow-up, he is completely free of the bleeding episodes without any medical intervention.

\section{Case 3}

A 9-year-old pre-pubertal girl presented to our

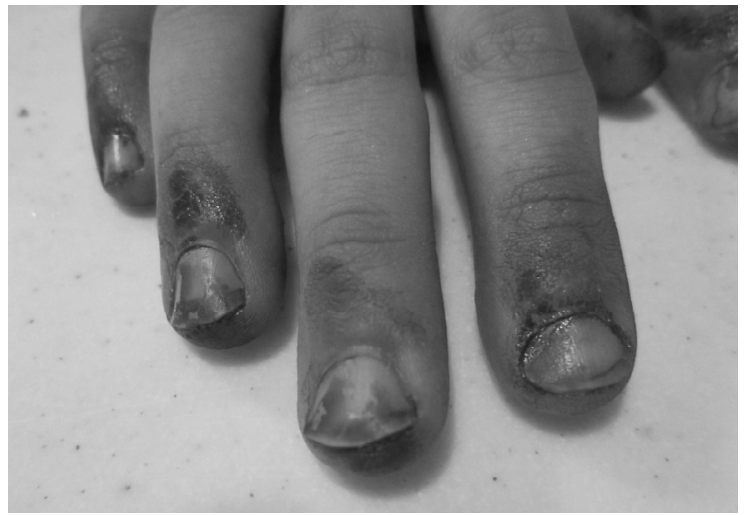

Fig. 2. Bloody secretion from the nail beds of an 8-yearold boy. 
clinic for bleeding disorder investigation. The bleeding was described to be spontaneous, unpredictable, and intermittent, with no specific patterns from her ears lobules, nose, eyes, and it stopped spontaneously. All investigations were found to be normal. Several episodes of bleeding occurred prior to admission, and one episode of bleeding from the ear lobules was observed by the medical personnel (Fig. 3 ). The secretion was moist, less viscous than blood and there was no break or lesion on the skin. The episode took about 5 minutes and ceased spontaneously. Hematohidrosis was considered and she was discharged on beta-blocker propranolol $10 \mathrm{mg}$ twice daily. She noticed a significant improvement in severity and frequency of the episodes. During 6 months follow-up, she did not experience any new episode.

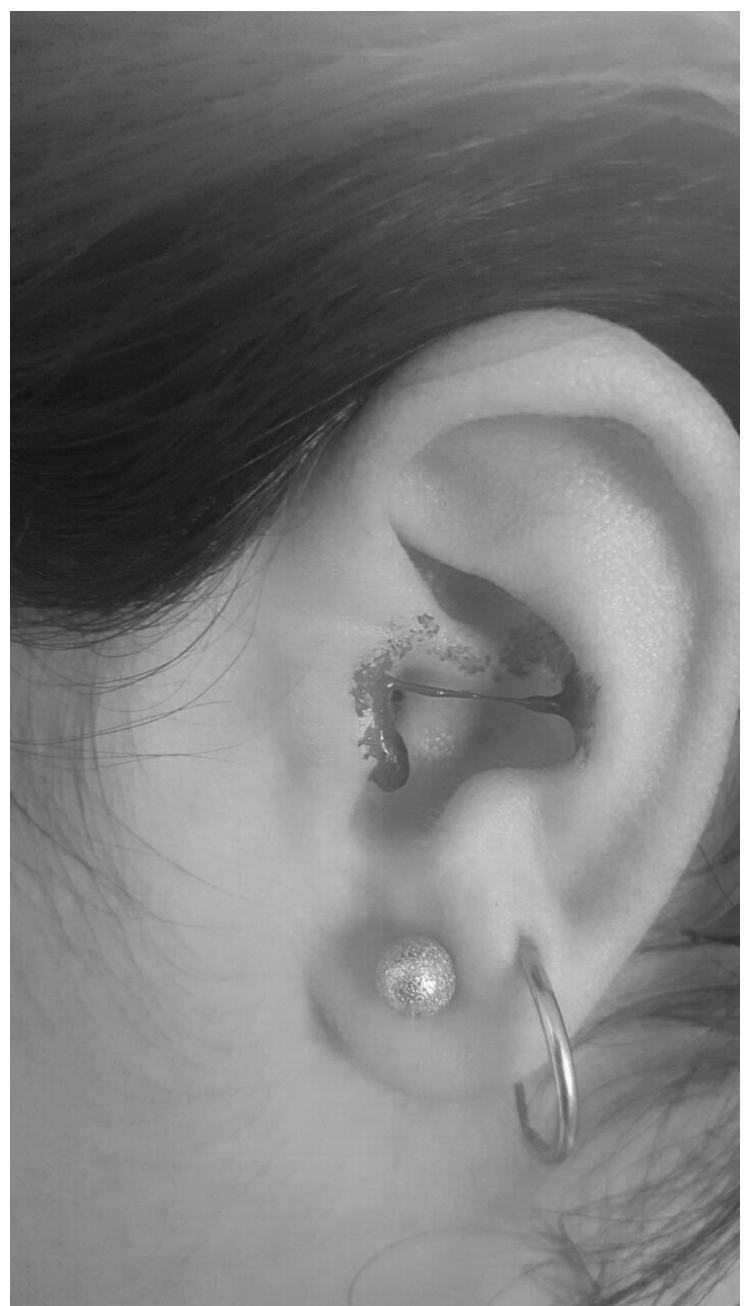

Fig. 3. Bloody secretion from the ears lobules of a 9-yearold pre-pubertal girl.

\section{Discussion}

Hematohidrosis, (bloody sweat), also known as hematidrosis, hemidrosis or hematofolliculohidrosis, is a very rare condition in which blood is exuded from intact skin surface or mucosa in almost any part of the body. It may occur at several points simultaneously. ${ }^{1}$ Despite being extremely rare and having no clear scientific evidence to support its existence, hematohidrosis is real and has been reported for many centuries throughout the world. Both religious and non-religious causes have been attributed to this condition. ${ }^{2}$ Interestingly, it has been tied to religious belief as stigmatization. The Bible mentions Jesus Christ experienced sweating tears of blood while praying before the crucifixion. Also, Leonardo Da Vinci, the prominent Renaissance artist and writer, described a soldier sweating blood before going to battle. A famous Italian physician in the 16th century Antonio Brassavola treated a nun who cried blood filled tears during her menstrual cycle. ${ }^{2}$

Diagnosis of hematohidrosis can only be made if the following criteria are met: (i) recurrent, spontaneous, painless and self-limited oozing of bloody discharge is witnessed and confirmed by health professionals, (ii) the usual blood components are found on biochemistry studies of the discharge, and (iii) the site of bleeding is intact with no abrasion, telangiectasia or purpura and after wiping the area, there is no evidence of oozing. All of these criteria must be met in order to rule out organic bleeding disorders, self-inflicted bleeding, factitious disorder by proxy, and chromhidrosis (colored sweat). ${ }^{1,3,4}$

The etiology and pathogenesis of hematohidrosis remain unclear. Although fear and anxiety seem to be the most commonly reported causes of hematohidrosis, it may happen without any preceding stressful situation. There is a classification system that includes five categories based on causative factors. ${ }^{1}$ These are systemic disease, vicarious menstruation, excessive exertion, psychogenic, and unknown. ${ }^{1}$ Epileptic seizures and Platelet Factor 3 dysfunction are also attributed to hematohidrosis. ${ }^{5,6}$ In our cases, there was no evidence of selfinjury and diagnosis was established with a demonstration of blood corpuscles in the secretion along with negative tests for bleeding 
diathesis. Vicarious menstruation (i.e. cyclical bleeding in extra-genital organs during a normal menstrual cycle) was ruled out in our two young female patients because they initially experienced bleeding episodes before menarche.

There are a number of theories that explain how hematohidrosis occurs. The general consensus relates to intensified sympathetic activation due to extreme physical or emotional stress. It is found that the sympathetic flight-or-fight response to intense stress leads to constriction of capillary vessels feeding the sweat glands. When the anxiety subsides, the blood vessels dilate to the point of rupture, leading to the passage of blood through the ducts of the nearby sweat glands and presenting as droplets of blood mixed with sweat on the intact skin surface or mucosa in almost any part of the body. Such manifestations may occur at several points simultaneously. ${ }^{2,7,8}$ Dermal vasculitis is also concluded as a pathological basis for hematohidrosis. ${ }^{9}$ Stromal weakness due to defects in the dermis is another theory to explain the occurrence of hematohidrosis. ${ }^{2}$ According to this theory, the communication between these defects and vascular spaces in the dermis may lead to the establishment of dilated blood centers. Whenever the positive pressure inside vascular spaces exceeds a certain level, blood will exude via follicular canals or directly into the skin surface. Subsequently, they will collapse and leave no scar. This phenomenon, which acts like a balloon, will wax and wane; thus explaining why the bleedings are intermittent and self-limiting. The bleeding is intermittent because the vascular spaces will disappear after exuding their content but then reoccurred after the blood flow is reestablished. ${ }^{2}$ An immediate biopsy is important for definite diagnosis. Biopsy during symptom- free period does not reveal any blood-filled vascular spaces, intradermal bleeding or abnormality in hair follicles and sebaceous or sweat glands. ${ }^{8}$ The term "hematofolliculohidrosis" is proposed as it appears along with sweat like fluid and the blood pushes via the follicular canals. ${ }^{2,9}$

The treatment of hematohidrosis still remains a challenge. There are some reports of successful use of propranolol ${ }^{10}$. Also, a trial of transdermal atropine has been shown to be effective in diminishing the severity and frequency of bloody discharge episodes in patients with a diagnosis of hematohidrosis ${ }^{3}$. In addition, in a case with simultaneous epileptic seizures and hematohidrosis, the symptoms of both were successfully resolved following the administration of the antiepileptic drug, oxcarbazepine. ${ }^{5}$ In our patients, beta-blocker therapy diminished the frequency of episodes.

In conclusion, despite its rarity, hematohidrosis should be considered as a miscellaneous differential diagnosis of bleeding episodes in a patient with normal physical and laboratory investigations. Such awareness can also play an important role in ensuring a non-judgmental and compassionate approach by physicians. This is an important issue because an overzealous rush to accuse parents of factitious disorder can have tragic consequences for a family that is seeking help for their child who suffers from this very rare disorder. Further studies need to search for etiology and risk factors of such condition to correctly address clinical management.

\section{Acknowledgements}

The author wishes to thank the Research Development Center of Bahrami Children Hospital.

\section{REFERENCES}

1. Holoubek JE, Holoubek AB. Blood, sweat and fear: A classification of hematidrosis. J Med 1996; 27: 115-133.

2. Manonukul J, Wisuthsarewong W, Chantorn R, Vongirad A, Omeapinyan P. Hematidrosis: A pathologic process or stigmata. A case report with comprehensive histopathologic and immunoperoxidase studies. Am J Dermatopathol 2008; 30: 135-139.

3. Biswas S, Surana T, De A, Nag F. A curious case of sweating blood. Indian J Dermatol 2013; 58: 478-480.

4. Bhagwat PV, Tophakhane RS, Rathod RM, Shashikumar BM, Naidu V. Hematohidrosis. Indian J Dermatol Venereol Leprol 2009; 75: 317-318.

5. Shen H, Wang Z, Wu T, et al. Haematidrosis associated with epilepsy in a girl successfully treated with oxcarbazepine: Case report. J Int Med Res 2015; 43: 263-269.

6. Mishra KL. Bloody tears and hematohidrosis in a patient of PF3 dysfunction: A case report. Cases J 2009; 2: 9029.

7. Jerajani HR, Jaju B, Phiske MM, Lade $\mathrm{N}$. Hematohidrosis-A rare clinical phenomenon. Indian J Dermatol 2009; 54: 290-292.

8. Zhang FK, Zhang YL, Liv JH, et al. Clinical and laboratory study of a case of hematidrosis. Zhonghua Xue Ye Xue Za Zhi 2004; 25: 147-150. 
9. Praveen BK, Vincent J. Hematidrosis and hemolacria: A case report. Indian J Pediatr 2012; 79: 109-111.
10. Wang Z, Yu Z, Su J, et al. A case of hematidrosis successfully treated with propranolol. Am J Clin Dermatol 2010; 11: 440-443. 